\title{
Cost-based Modeling for Fraud and Intrusion Detection: Results from the JAM Project
}

\author{
Salvatore J. Stolfo, Wei Fan \\ Computer Science Department \\ Columbia University \\ 500 West 120th Street, New York, NY 10027 \\ $\{$ sal,wfan\}@cs.columbia.edu
}

\author{
Wenke Lee \\ Computer Science Department \\ North Carolina State University \\ Raleigh, NC 27695-7550 \\ wenke@csc.ncsu.edu
}

\author{
Andreas Prodromidis \\ iPrivacy \\ 599 Lexington Ave., \#2300, New York, NY 10022 \\ andreas@iprivacy.com \\ Philip K. Chan \\ Computer Science Department \\ Florida Institute of Technology \\ 150 W. University Blvd., Melbourne, FL 32901 \\ pkc@cs.fit.edu
}

\begin{abstract}
In this paper we describe the results achieved using the JAM distributed data mining system for the real world problem of fraud detection in financial information systems. For this domain we provide clear evidence that state-of-the-art commercial fraud detection systems can be substantially improved in stopping losses due to fraud by combining multiple models of fraudulent transaction shared among banks. We demonstrate that the traditional statistical metrics used to train and evaluate the performance of learning systems, (i.e. statistical accuracy or ROC analysis) are misleading and perhaps inappropriate for this application. Cost-based metrics are more relevant in certain domains, and defining such metrics poses significant and interesting research questions both in evaluating systems and alternative models, and in formalizing the problems to which one may wish to apply data mining technologies.

This paper also demonstrates how the techniques developed for fraud detection can be generalized and applied to the important area of Intrusion Detection in networked information systems. We report the outcome of recent evaluations of our system applied to tcpdump network intrusion data specifically with respect to statistical accuracy. This work involved building additional components of JAM that we have come to call, MADAM ID (Mining Audit Data for
\end{abstract}

Automated Models for Intrusion Detection). However, taking the next step to define cost-based models for intrusion detection poses interesting new research questions. We describe our initial ideas about how to evaluate intrusion detection systems using cost models learned during our work on fraud detection.

\section{Introduction}

In this paper we discuss the results achieved over the past several years on the JAM Project ${ }^{1}$. JAM stands for Java Agents for Meta-Learning. JAM was initiated as a DARPA and NSF sponsored research project studying algorithms, techniques and systems for distributed data mining. The initial conceptions that we proposed involves the use of agentbased technologies to dispatch machine learning and data analysis programs to remote database sites, resulting in a distributed collection of derived models $[4,5,6,25]$. Subsequently, the same agent-based technology provides the means for derived "base" models to migrate in a network information system and be collected at any of the participating sites where they may be combined. A single "aggregate"

\footnotetext{
${ }^{1}$ Browse http://www.cs.columbia.edu/ sal/JAM/ PROJECT for the complete set of progress reports, publications and JAM software available for download.
} 
model would then be computed by combining the remotely computed base models, by a technique we have called "meta-learning", that outputs a "meta-classifier". Each parallel site may compute its own meta-classifier, trained on locally stored data. These meta-classifiers likewise can migrate around the network to be combined with each other in a hierarchical fashion. The architecture of JAM has been reported extensively in prior papers [24, 26].

JAM's utility has been demonstrated in the context of real-world problems that in and of themselves are very important. Fraud and intrusion detection are key elements of a new national challenge to protect our nation's critical infrastructures.

\section{The Fraud Detection Problem}

We consider the problem of detecting fraudulent transactions after they have been subject to fraud prevention methods and processes. There is a vast literature on various security methods to protect transactors from unauthorized use or disclosure of their private information and valuable assets.

Financial institutions today typically develop custom fraud detection systems targeted to their own asset bases. The key concept in fraud detection is that fraud may be detected by noticing significant deviation from the "normal behavior" of a customer's account. The behavior of an account can thus be used to protect that account. Notice, it is considerably easier to steal someone's identity information than it is to steal their behavior ${ }^{2}$.

Recently though, banks have come to realize that a unified, global approach is required to detect fraud, involving the periodic sharing with each other of information about attacks. We have proposed a new wall of protection consisting of pattern-directed inference systems using models of fraudulent transaction behaviors to detect attacks. This approach requires analysis of large and inherently distributed databases of information about transaction behaviors to produce models of "probably fraudulent" transactions. We have used JAM to compute these models.

JAM is used to compute local fraud detection agents that learn how to detect fraud and provide intrusion detection services within a single information system; JAM provides an integrated meta-learning system that combines the collective knowledge acquired by individual local agents from among participating bank sites. Once derived local classifier agents or models are produced at some datasite(s), two or more such agents may be composed into a new classifier agent by JAM's meta-learning agents. JAM allows financial institutions to share their models of fraudulent transactions

\footnotetext{
${ }^{2}$ This may change as vast databases of "click data" revealing personal behavior information continues to be acquired over the Internet typically without the user's knowledge.
}

by exchanging classifier agents in a secured agent infrastructure. But they will not need to disclose their proprietary data. In this way their competitive and legal restrictions can be met, but they can still share information. The metaclassifiers then act as sentries forewarning of possibly fraudulent transactions and threats by inspecting, classifying and labeling each incoming transaction.

\subsection{The Fraud Learning Task}

The learning task here is quite straightforward. Given a set of "labeled transactions", $T=\left\{t \mid t=<f_{1}, \ldots, f_{n}>\right\}$, compute a model or classifier, $C$, by some learning algorithm $L$, that predicts from the features $<f_{1}, \ldots, f_{n-1}>$ the target class label $f_{n}$, "fraud" or "legitimate". Hence, $C=L(T)$, where $L$ is a learning algorithm. Each element $t \in T$ is a vector of features, where we denote $f_{1}$ as the "transaction amount" (tranamt), and $f_{n}$ as the target class label, denoted $\operatorname{fraud}(t)=0$ (legitimate transaction) or 1 (a fraudulent transaction). Given a "new unseen" transaction, $x$, with unknown class label, we compute $f_{n}(x)=C(x)$. $C$ serves as our fraud detector.

Much of our work on the JAM project has focussed on developing, implementing and evaluating a range of learning strategies and combining techniques. Our work on "meta-learning" strategies has focussed on computing sets of "base classifiers" over various partitions or samplings of the training data, $T$, and various performance metrics to evaluate base classifiers over test data. (Nearly all of our reported results are based upon k-fold cross validation.)

In meta-learning, we first seek to compute a set of base classifiers, $\left\{C_{i}, i=1, \ldots, m\right\}$, where $C_{i}=L_{j}\left(T_{k}\right)$, $\bigcup_{k} T_{k}=T$, varying the distributions of training data $\left(T_{k}\right)$ and using a variety of different machine learning algorithms $\left(L_{j}\right)$ in order to determine the "best" strategies for building good fraud detectors. The "best" base classifiers are then combined by a variety of techniques in order to boost performance. One of the simplest combining algorithms proposed in[3], and independently by Wolpert[29], is called "class-combiner" or "stacking". A separate hold out training dataset, $V$, is used to generate a meta-level training data to learn a new "meta-classifier" $M . M$ is computed by learning a classifier from training data composed of the predictions of a set of base classifiers generated over a set of validation data $(V)$ along with the true class label. Hence, $M=L\left(<C_{1}(v), \ldots, C_{m}(v), f_{n}(v)>\right), v \in V$. The resultant meta-classifier works by inputing the predictions for some unknown into its constituent base classifiers, and then generating its own final class prediction from these base classifier predictions. Thus, for unknown $x, f_{n}(x)=M\left(C_{1}(x), \ldots, C_{m}(x)\right)$.

Notice, $M$ is as well a classifier, or fraud detector. In the following sections when we make reference to a classi- 
fier $C$, it may either be a "base classifier", or an ensemble "meta-classifier", learned over some training distribution, unless explicitly stated otherwise.

For notational convenience, we define $J=$ $F\left[L, M, T, D_{T}\right]$ as the set of all classifiers that may be computed where $L=\left\{L_{1}, \ldots, L_{p}\right\}$ is a set of learning algorithms, $M\left\{M_{1}, \ldots, M_{q}\right\}$ is a set of meta-learning algorithms, $T$ is a set of labeled ground truth data used to train classifiers, and $D_{T}$ is some training distribution of interest. JAM is a computational environment that is designed to assist data miners in generating desired classifiers.

\subsection{Credit Card Datasets}

Chase and First Union Banks, members of the FSTC (Financial Services Technology Consortium) provided us with real credit card data for our studies. The two data sets contain credit card transactions labeled as fraudulent or legitimate. Each bank supplied .5 million records spanning one year with $20 \%$ fraud and $80 \%$ non-fraud distribution for Chase bank and $15 \%$ versus $85 \%$ for First Union bank. The schemata (or feature sets) of the databases were developed over years of experience and continuous analysis by bank personnel to capture important information for fraud detection. We cannot reveal the details of the schema beyond what is described in [19]. The records have a fixed length of 137 bytes each and about 30 attributes including the binary class label $\left(f_{n}\right)$. Some of the fields are numeric and the rest categorical, i.e. numbers were used to represent a few discrete categories.

The features in this data defined by the banks essentially describe the "usage behavior" of an individual credit card account. The data is rich enough to allow alternative modeling strategies. For example, it is possible to segment the data into classes of accounts based upon "payment history" 3 . It is well known that there are at least two classes of card holders, "transactors" who pay their bills in full each month, and "revolvers" who pay their minimum charge and roll over balances and interest charges to the next month. Models can thus be built for each of these market segments to determine finer distinctions between account transaction behaviors. In this work, we do no such segmentation and partitioning, but rather we compute models of "fraudulent transaction" for the entire asset base. We believe the results achieved in this study can be improved had we segmented

\footnotetext{
${ }^{3}$ The reader is encouraged to do a personal study of their own checking account behavior. You will likely find the same type of payments from month to month in your own checking account. Variations or distinct payments in any particular month are likely to reoccur in the same month in the prior year. Such repetitive behaviors can be regarded as a normal profile for the checking account. Significant variations from this normal profile possibly indicate fraud.
}

the data ${ }^{4}$.

Many experiments were conducted using JAM to evaluate the performance of different learning algorithms, and different meta-level training sets using this data. Prior publications report on these experiments and indicate that the meta-classifiers consistently outperform the best base classifiers. Unfortunately, the studies that considered different training distributions, different learning algorithms and a variety of combining techniques demonstrated that the best strategies for this target domain are not immediately discernible, but rather requires extensive experimentation to find the best models, and the best meta-classifiers [20].

\subsection{Cost-based Models for Fraud Detection}

Most of the machine learning literature concentrates on model accuracy (either training error or generalization error on hold out test data computed as overall accuracy, True Positive/False Positive rates, or ROC analysis). This domain provides a considerably different metric to evaluate performance of learned models; models are evaluated and rated by a "cost model." Within the context of financial transactions, cost is naturally measured in dollars. However, any unit of measure of utility applies here. The credit card domain provides an excellent motivating example domain familiar to most people.

Due to the different dollar amounts of each credit card transaction and other factors, the cost of failing to detect a fraud varies with each transaction. Hence, the cost model for this domain is based on the sum and average of loss caused by fraud. We define for a set of transactions $S$, a fixed overhead amount, and a fraud detector (or classifier) $C$ :

$$
\begin{gathered}
\text { Cumulative } \operatorname{Cost}(S, C, \text { overhead })= \\
\sum_{t \in S}^{n} \operatorname{Cost}(C(t, \text { overhead }))
\end{gathered}
$$

and

$$
\begin{aligned}
& \text { AverageCost }(S, C, \text { overhead })= \\
& \qquad \frac{\text { CumulativeCost }(S, C, \text { overhead })}{n}
\end{aligned}
$$

where Cost $(t$, overhead $)$ is the cost associated with transaction $t$ and $n$ is the total number of transactions in a test set $S$. The cost of a transaction is not simply its "transaction amount", but is also a function of an overhead amount.

After consulting with a bank representative, we jointly settled on a simplified cost model that closely reflects reality. Since it takes time and personnel to investigate a potential fraudulent transaction, a fixed overhead value is incurred for each investigation. That is, if the amount of a

\footnotetext{
${ }^{4}$ However, such a strategy would delve dangerously close to industry trade secrets.
} 


\begin{tabular}{lll}
\hline Outcome & Cost $(\mathrm{t}$, overhead) & \\
\hline Miss (False Negative, $F N)$ & tranamt $(t)$ & \\
False Alarm (False Positive, $F P)$ & overhead & if tranamt $(t)>$ overhead \\
& 0 & if $\operatorname{tranamt}(t) \leq$ overhead \\
Hit (True Positive, $T P)$ & overhead & if $\operatorname{tranamt}(t)>$ overhead \\
& tranamt $(t)$ & if $\operatorname{tranamt}(t) \leq$ overhead \\
Normal (True Negative, $T N)$ & 0 & \\
\hline
\end{tabular}

Table 1. Cost Model for Transaction

transaction is smaller than the overhead, it is not worthwhile to investigate the transaction even if it is suspicious. For example, if it takes ten dollars to investigate a potential loss of one dollar, it is more economical not to investigate. Assuming a fixed overhead, we devised the cost model for each transaction $\mathrm{t}$ and classifier $\mathrm{C}$, showed in Table 1. (Recall, $f_{1}(t)=\operatorname{tranamt}(t)$.) The overhead threshold, for good reason, is a closely guarded secret for important reasons discussed later in section 5.5, and may vary over time. The range of values used in our studies is probably reasonable as appropriate bounds for the data set provided by the banks. All the empirical studies we conducted are evaluated using this cost model.

It is important to note that this overhead is not a "score threshold" for classifiers that may output continuous values (i.e., density estimators). Rather, the threshold is a simple "decision boundary". Transactions whose amounts are under this threshold are immediately authorized (subject to account credit availability of course). Decisions are therefore made by detectors only for transactions above this threshold $^{5}$.

The target application is described as detecting frauds to minimize cumulative cost, or maximize cost savings in this model. In describing our results, we report the maximum savings (or stop loss in bank parlance), as the total dollar amount saved from detection under this cost model. The total potential dollar loss for a (test) set of transactions $(S)$ is defined as the total dollar amount of all fraudulent transactions:

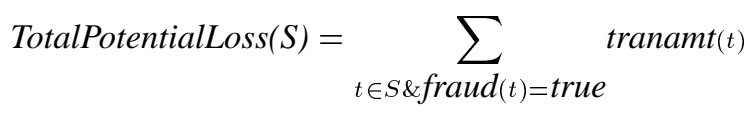

A complete comparative evaluation between purely statistical error rates versus cost-model savings can be found in

\footnotetext{
${ }^{5}$ When training classifiers, one may think that simply ignoring all transactions in the training data under the threshold will produce better detectors. This may not be true. The fraudulent transaction behavior we seek to learn may not vary with the transaction amount (thieves will do the same things whether stealing ten dollars, or a hundred dollars), and the learning of fraudulent behavior may be more accurate when studying low cost transactions. Even so, some experiments were performed varying the underlying distributions, and we describe these later.
}

the cited papers. The important lesson here is that the data mining problem is actually a straightforward cost optimization problem, namely to capture or recover the TotalPotentialLoss due to fraud. That is, given a test set of transactions, $S$, a fraud model $C_{\text {overhead }}$, and the overhead, the TotalCostSavings is defined as:

$$
\begin{gathered}
\text { TotalCostSavings }\left(S, C_{\text {overhead }}, \text { overhead }\right)= \\
\text { TotalPotentialLoss }(S)- \\
\text { CumulativeCost }\left(S, C_{\text {overhead }}, \text { overhead }\right)
\end{gathered}
$$

We can now state concretely what the learning task is for fraud detection. We seek to compute the

$$
\underset{C_{\text {overhead }} \in J}{\operatorname{argmax}}\{\text { TotalCostSavings }(S, C, \text { overhead })\}
$$

where individual classifiers, $C_{\text {overhead }}$, can be models computed (by JAM) over training data under the cost model with a fixed overhead, $T$, by many different learning and meta-learning strategies. (Note, the worst possible outcome is that the detector is so bad we actually lose money; the total cost savings may be negative.) We may of course add additional constraints on $C_{\text {overhead }}$ so that it, for example, meets real-time, or memory constraints, or is computed over inherently distributed data (partitions of $T$ ) as rapidly as possible. Explorations of these issues have been much of the focus of the JAM project.

It is interesting to note here another reason why pure accuracy measures are inappropriate in some domains. Observe in this and related domains (like cellular phone fraud) the distribution of frauds may constitute a very tiny percentage of the total number of transactions. If, for example, the percentage of frauds is $1 \%$ or less, than the null detector will be $99 \%$ accurate or better! Naturally, we must depend upon at least the TP/FP rates of a detector to measure alternatives. But, more to the point, training detectors in such domains begs an important question: what is the appropriate distribution of data used in training to produce "good detectors"? In our prior work many experiments were conducted to evaluate classifier performance over different samplings and distributions. For example, in one set of tests, 50:50 distribu- 
tions produced the best detectors ${ }^{6}$. Of particular note is that the best training distributions varied according to the overhead and thus the cost-model. This is why we subscript the classifier $C_{\text {overhead }}$ to reveal that alternative classifiers can be computed simply by varying this quantity. Here again, determining the best training distribution under realistic environments, with possibly highly skewed distributions is a matter of considerable empirical investigation.

\section{Results using JAM}

A large number of experiments were performed to determine whether various alternative training strategies would produce improved or superior models. The strategies included different temporal distributions (different months of training data), different partitions or samples (random, or different distributions of fraud versus non-fraud) and different cost distributions (training over large frauds versus lower cost frauds). The results we report here are only one broad view of what we achieved using the best strategies determined empirically as compared to the best possible outcome for a commercial off the shelf system.

CHASE provided us with data that had embedded within each transaction record a field recording a score (in the range of 0-999) generated by a commercial off-the-shelf (COTS) fraud detection system for that transaction. From this information we were able to easily compute the best possible detection cost savings of this system as well as its statistical accuracy. We do not know what the actual score thresholds may be in practice. However, for the same data provided by CHASE we can compute what the optimal setting for the COTS should be for optimal performance. 7 Using this, we were able to compare JAM's models to see if we were indeed doing better.

\subsection{Baseline Performance Under the Cost Model}

Tables 2 and 3 summarize our results for the Chase and First Union banks respectively. Both tables display the accuracy, the $T P-F P$ spread and savings for each of the fraud predictors examined. Recall, overall accuracy is simply the percentage of correct predictions of a classifier on a test set of "ground truth". TP means the rate of predicting "true positives" (the ratio of correctly predicted frauds over all of the true frauds), FP means the rate of predicting "false positives" (the ratio of incorrectly predicted frauds over those test examples that were not frauds, otherwise known as the "false alarm rate".) We use " $T P-F P$ spread" to indicate

\footnotetext{
${ }^{6}$ In this context, data is plentiful, so we could afford to construct many alternative distributions without much fear of generating "knowledge poor" training samples.

${ }^{7}$ We computed the performance of the COTS for a whole range of score thresholds: 5, 10, 20, 30, 40, 50, 100, 150, 200, 250, ... , 850, 800, 950.
}

how well the system finds true frauds versus false alarms. A "1.00 TP - FP spread" is optimal performance. ${ }^{8}$ The maximum loss potential of these test sets is approximately $\$ 1,470,000$ for the Chase data and $\$ 1,085.000$ for the First Union data. The column denoted as "size" indicates the number of base-classifiers used in the meta-classifier.

\subsection{JAM versus COTS}

The first row of Table 2 shows the best possible performance of Chase's own COTS authorization/detection system on this data set. The next two rows present the performance of the best base classifiers over the entire set and over a single month's data, while the last rows detail the performance of the unpruned (size of 50) meta-classifiers. Similar data is recorded in Table 3 for the First Union set, with the exception of First Union's COTS authorization/detection performance (it was not made available to us).

The outcome was clearly in favor of JAM for this dataset. According to these results, the COTS system achieves $85.7 \%$ overall accuracy, 0.523 " $T P-F P$ spread" and saves $\$ 682 \mathrm{~K}$ when set to its optimal "score threshold".

A comparison of the results of Tables 2 and 3 indicates that in almost all instances, meta-classifiers outperform all base classifiers, and in some cases by a significant margin. The most notable exception is found in the "savings" column of Chase bank where the meta-classifier exhibits reduced effectiveness compared to that of the best base classifier.

This shortcoming can be attributed to the fact that the learning task is ill-defined. Training classifiers to distinguish fraudulent transactions is not a direct approach to maximizing savings (or the $T P-F P$ spread). Traditional learning algorithms are not biased towards the cost model and the actual value (in dollars) of the fraud/legitimate label; instead they are designed to minimize statistical misclassification error. Hence, the most accurate classifiers are not necessarily the most cost effective. Similarly, the metaclassifiers are trained to maximize the overall accuracy not by examining the savings in dollars but by relying on the predictions of the base-classifiers. Naturally, the metaclassifiers are trained to trust the wrong base-classifiers for the wrong reasons, i.e. they trust the base classifiers that are most accurate instead of the classifiers that accrue highest savings.

\subsection{Bridging Classifiers for Knowledge Sharing}

The final stage of our experiments on the credit card data involved the exchange of base classifiers between the two

\footnotetext{
${ }^{8}$ These are standard terms from the statistical "confusion" matrix that also includes FN and TN rates as well.
} 


\begin{tabular}{|l|c||c|c|c|}
\hline Type of Classification Model & Size & Accuracy & TP - FP & Savings \\
\hline \hline COTS scoring system from Chase & - & $85.7 \%$ & 0.523 & $\$ 682 \mathrm{~K}$ \\
\hline Best base classifier over one subset & 1 & $88.5 \%$ & 0.551 & $\$ 812 \mathrm{~K}$ \\
\hline Best base classifier over entire set & 1 & $88.8 \%$ & 0.568 & $\$ 840 \mathrm{~K}$ \\
\hline Meta-classifier, Chase & 50 & $89.6 \%$ & 0.621 & $\$ 818 \mathrm{~K}$ \\
\hline
\end{tabular}

Table 2. Performance results for the Chase credit card data set.

\begin{tabular}{|l|c||c|c|c|}
\hline Type of Classification Model & Size & Accuracy & TP - FP & Savings \\
\hline \hline Best base classifier over one subset & 1 & $95.2 \%$ & 0.749 & $\$ 806 \mathrm{~K}$ \\
\hline Best base classifier over entire set & 1 & $95.3 \%$ & 0.787 & $\$ 828 \mathrm{~K}$ \\
\hline Meta-classifier, First Union & 50 & $96.5 \%$ & 0.831 & $\$ 944 \mathrm{~K}$ \\
\hline
\end{tabular}

Table 3. Performance results for the First Union credit card data set.

banks. To meta-learn over a set of classifier agents, however, we had to overcome additional obstacles in order to share their knowledge of fraud. The two databases had differences in their schema definition of the transactions, and hence learning over these different sites produced incompatible classifiers:

1. Chase and First Union defined an attribute with different semantics (i.e. one bank recorded the number of times an event occurs within a specific time period while the second bank recorded the number of times the same event occurs within a different time period).

2. Chase includes two (continuous) attributes not present in the First Union data.

To address these problems we followed the approaches described in $[14,21,18]$. For the first incompatibility, we had the values of the First Union data set mapped via a linear approximation to the semantics of the Chase data. For the second incompatibility, we deployed special bridging agents that were trained to compute the missing values of First Union data set. The training involved the construction of regression models [23] of the missing attributes over the Chase data set using only the attributes that were common to both banks. When predicting, the First Union classifier agents simply disregarded the real values provided at the Chase data sites, while the Chase classifier agents relied on both the common attributes and the predictions of the bridging agents to deliver a prediction at the First Union data sites.

Tables 4 and 5 display the accuracy, $T P-F P$ spread and cost savings of each Chase and First Union meta-classifier. These results demonstrate that both Chase and First Union fraud detectors can be exchanged and applied to their respective data sets. The most apparent outcome of these experiments is the superior performance of the First Union meta-classifiers and the lack of improvement on the performance of the Chase meta-classifiers This phenomenon can be easily explained from the fact that the attributes missing from the First Union data set were significant in modeling the Chase data set. Hence, the First Union classifiers are not as effective as the Chase classifiers on the Chase data, and the Chase classifiers cannot perform at their best at the First Union sites without the bridging agents. The latter was verified by a separate experiment, similar to the above, with the exception that no bridging agents were used, i.e. Chase classifiers produced predictions without using any information on the missing values.

The bottom line is that our hypothesis was correct: better performance resulted from combining multiple fraud models by distributed data mining over different transaction record sources (including multiple banks) even when bridging the differences among their schema.

\subsection{Cost-sensitive Learning: AdaCost}

Much of our experimental work has been to "bias" the outcome of the learned classifiers towards improved cost performance by varying training distributions, or pruning poor cost performing classifiers. This approach is somewhat akin to hammering a square peg into a round hole.

An alternative strategy is called cost sensitive learning. The essence of the idea is to bias feature selection in generating hypotheses during the learning process in favor of those that maximize a cost criterion (for example, the cost of testing features, rather than a purely statistical criterion). According to Turney [28] the earliest work here is due to Nunez [17]. Later work by Tan and Schlimmer [27] also incorporates feature costs in the heuristic for searching in a modified decision tree learning algorithm. However, there are costs associated not only with testing features, but also varying costs based upon classifier misclassification cost performance. The distinctions are important.

Two alternative features may have the same "test cost" but their predictive outcomes may produce different "misclassification costs." Hence, we ought to strategically 


\begin{tabular}{|l|c||c|c|c|}
\hline Composition of Meta-Classifier & Size & Accuracy & TP - FP & Savings \\
\hline \hline Chase + First Union & 110 & $89.7 \%$ & 0.621 & $\$ 800 \mathrm{~K}$ \\
\hline Chase + First Union & 63 & $89.7 \%$ & 0.633 & $\$ 877 \mathrm{~K}$ \\
\hline
\end{tabular}

Table 4. Combining Chase and First Union classifiers on Chase data.

\begin{tabular}{|l|c||c|c|c|}
\hline Composition of Meta-Classifier & Size & Accuracy & TP - FP & Savings \\
\hline \hline First Union + Chase - bridge & 110 & $96.6 \%$ & 0.843 & $\$ 942 \mathrm{~K}$ \\
\hline First Union + Chase + bridge & 110 & $97.8 \%$ & 0.901 & $\$ 963 \mathrm{~K}$ \\
\hline First Union + Chase + bridge & 56 & $97.8 \%$ & 0.899 & $\$ 962 \mathrm{~K}$ \\
\hline
\end{tabular}

Table 5. Combining Chase and First Union classifiers on First Union data.

choose "low cost features" that are both cheap to compute and test, and that reduce the misclassification cost of the final model that employs them.

What the cost model for the credit card domain teaches is that there are different costs depending upon the outcome of the predictions of the fraud detectors. This may appear strange but we may want to compute classifiers that are purposely wrong in certain cases so that we do not incur their high costs when they predict correctly. Not only are there costs associated with "misclassifications" (False positives/negatives), but also costs are born with Correct Predictions, i.e. True Positives also incur costs (overhead)! This simple, but perhaps counterintuitive, this observation has not been accounted for in prior work and has been included in our cost models when computing classifiers and evaluating their outcome.

As mentioned, we have performed experiments to generate cost-sensitive classifiers by varying the distribution of training examples according to their costs (tranamt). This strategy doesn't change the underlying algorithm, but rather attempts to bias the outcome of the underlying (statisticalbased) algorithm.

This was achieved by two simple methods: replication and biased sampling. In the first case, experiments were performed where training data was "replicated" some number of times based upon the cost of the exemplars. Another strategy sampled high cost examples and excluded the low cost transactions (those under the overhead amount). These "cost-based training distributions" were used in training base models, and meta-classifiers. Unfortunately, the results indicated that the resultant classifiers did not consistently improve their cost performance [7] over varying cost distributions.

Other experiments were performed to directly bias the internal strategy of the learning algorithm. One algorithm we have proposed and studied is a close variant of Singer and Schapire's [22] AdaBoost algorithm. AdaBoost is an algorithm that starts with a set of "weak hypotheses" of some training set, and iteratively modifies weights associ- ated with these hypotheses based upon the statistical performance of the hypotheses on the training set. Elements of the training set are as well weighted, and updated on successive rounds depending upon the statistical performance of the hypotheses over the individual data elements. AdaBoost ultimately, therefore, seeks to generate a classifier with minimum training error.

AdaCost [9] is a variant of AdaBoost that modifies its "weight updating rule" by a "cost based factor" (a function of tranamt and the overhead). Here, training elements that are "misclassified" are re-weighted by a function of the statistical performance of the hypotheses as well as the "cost" of the element. Costlier misclassifications are "reweighted" more for training on the next round. All weights are normalized on each round so correct predictions have their weights reduced. However, the new weights of correct predictions are adjusted by the cost model to account for the cost of true positives as well.

It is not possible to change the underlying training distribution according to the credit card cost model because the cost of a transaction is dependent upon the final prediction of the classifier we are attempting to compute, and is not known a priori, i.e., during training. Since the credit card cost model dictates cost even if the classification is correct, adjusting weights of training examples can't easily reflect that fact. The best we can do here is incorporate the cost for correct predictions on the "current round" during training to produce a different distribution for the "next round" of training.

Experiments here using AdaCost on the credit card data showed consistent improvement in "stopping loss" over what was achieved using the vanilla AdaBoost algorithm. For example, the results plotted in Figure 1 shows the average reduction of 10 months as a percentage cumulative loss (defined as $\frac{\text { cumulative loss }}{\text { maximal loss-least loss }} * 100 \%$ ) for AdaBoost and AdaCost for all 50 rounds and 4 overheads. We can clearly see that, except for round 1 with overhead $=90$, there is a consistent reduction for all other $398(=50 * 2 * 4-2)$ runs. The absolute amount of reduction is around $3 \%$. 

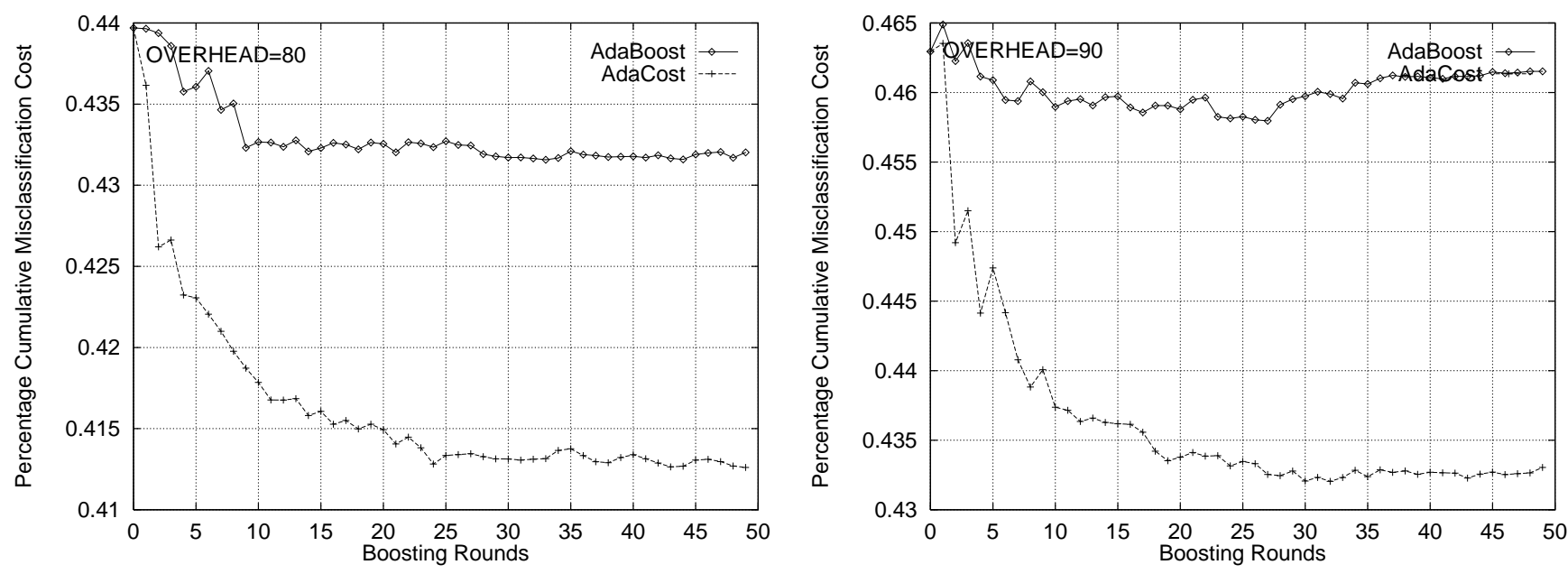

Figure 1. Cumulative Loss Ratio of AdaCost and AdaBoost for Chase Credit Card Data Set

We also observe that the speed of reduction by AdaCost is quicker than that of AdaBoost. Figure 2 plots the ratio of cumulative cost by AdaCost and AdaBoost. We have plotted the results of all 10 pairs of training and test months over all rounds and overheads. Most of the points are below the "Ratio=1" line in the left drawing and above the " $\mathrm{y}=\mathrm{x}$ " line in the plot on the right, both implying that AdaCost has lower cumulative loss in an overwhelming number of cases.

\section{Intrusion Detection: Initial results using MADAM ID}

Encouraged by our results in fraud detection ${ }^{9}$, we shifted our attention to the growing problem of intrusion detection in network based systems. Here the problems are significantly different, although from a certain perspective we seek to perform the same sort of task as in the credit card fraud domain. We seek to build models of "normal" behavior to distinguish between "bad" (intrusive) connections and "good" (normal) connections.

MADAM ID (Mining Audit Data for Automated Models for Intrusion Detection) is a set of new data mining algorithms that were developed by our project specifically to process network intrusion and audit data sets. MADAM ID includes variants of the "association rule" $[1,2]$ and "frequent episodes" $[16,15]$ algorithms used to define new feature sets that are extracted from labeled tcpdump data in order to define training sets for a machine learning algorithm to compute detectors. These features are defined over a set of connections. We first determine what patterns of events in the raw stream appear to occur frequently in attack connections that do not appear frequently in normal connections. These patterns of events define "features" computed

\footnotetext{
${ }^{9}$ And under "encouragement" from DARPA
}

for all connections used in training a classifier by some inductive inference or machine learning algorithm. The details of this data mining activity have been extensively reported [10,12]. (Our previous exploratory work on learning anomalous Unix process execution traces can be found in [11].) Here we report a summary of our results.

\subsection{The DARPA/MIT Lincoln Lab ID Evaluation}

We participated in the 1998 DARPA Intrusion Detection Evaluation Program, prepared and managed by MIT Lincoln Lab. The objective of this program was to survey and evaluate research in intrusion detection. A standard set of extensively gathered audit data, which includes a wide variety of intrusions simulated in a military network environment, was provided by DARPA. Each participating site was required to build intrusion detection models or tweak their existing system parameters using the training data, and send the results (i.e., detected intrusions) on the test data back to DARPA for performance evaluation.

We were provided with about 4 gigabytes of compressed raw (binary) tcpdump data of 7 weeks of network traffic, which can be processed into about 5 million connection records, each with about 100 bytes. The two weeks of test data have around 2 million connection records. Four main categories of attacks were simulated: DOS, denial-ofservice, e.g., syn flood; R2L, unauthorized access from a remote machine, e.g., guessing password; U2R, unauthorized access to local superuser (root) privileges, e.g., various of "buffer overflow" attacks; and PROBING, surveillance and probing, e.g., port-scan.

Using the procedures reported in prior papers [12] we compared the aggregate normal pattern set with the patterns from each dataset that contains an attack type. The following features were constructed according to the intrusion 

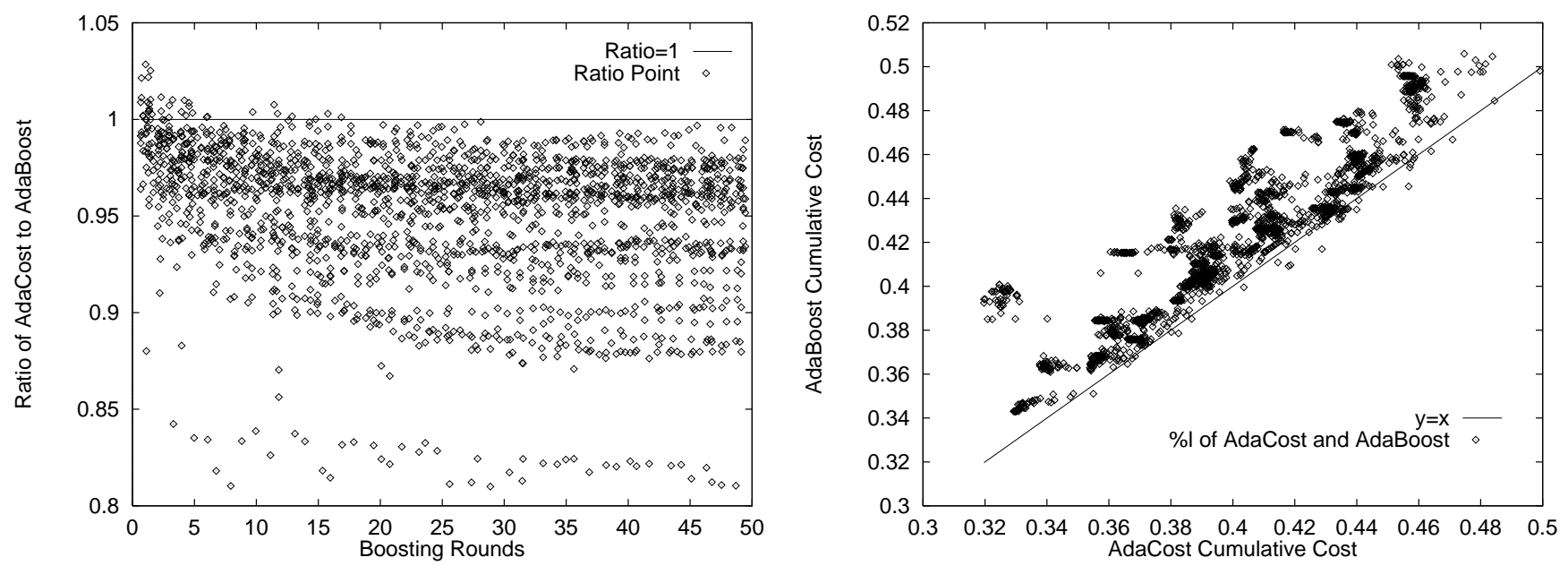

Figure 2. Cumulative Loss Ratio and Loss of AdaCost and AdaBoost on Chase Credit Card

only patterns:

- The "same host" features that examine only the connections in the past 2 seconds that have the same destination host as the current connection, and calculate statistics related to protocol behavior, service, etc.

- The similar "same service" features that examine only the connections in the past 2 seconds that have the same service as the current connection.

We call these the (time-based) "traffic" features of the connection records. There are several "slow" PROBING attacks that scan the hosts (or ports) using a much larger time interval than 2 seconds, for example, one in every minute. As a result, these attacks did not produce intrusion only patterns with a time window of 2 seconds. We sorted these connection records by the destination hosts, and applied the same pattern mining and feature construction process. Rather than using a time window of 2 seconds, we now used a "connection" window of 100 connections, and constructed a mirror set of "host-based traffic" features as the (time-based) "traffic" features.

We discovered that unlike most of the DOS and PROBING attacks, the R2L and U2R attacks don't have any "intrusion only" frequent sequential patterns. This is because the DOS and PROBING attacks involve many connections to some host(s) in a very short period of time, the R2L and PROBING attacks are embedded in the data portions of the packets, and normally involves only a single connection. Algorithms for mining the unstructured data portions of packets are still under development. Presently, we use domain knowledge to add features that look for suspicious behavior in the data portion, e.g., number of failed login attempts. We call these features the "content" features.

We then built three specialized models, using RIPPER [8]. RIPPER is a rule learning program that outputs a model quite similar in style to a Prolog program. Each model produced has a different set of features and detects different categories of intrusions. For example, for the "content" model, each connection record contains the "intrinsic" features and the "content" features, and the resultant RIPPER rules employing these features detects $\mathrm{U} 2 \mathrm{R}$ and $\mathrm{R} 2 \mathrm{~L}$ attacks. A meta-classifier was trained and used to combine the predictions of the three base models when making a final prediction to a connection record. Table 6 summarizes these models. The numbers in bold, for example, 9, indicate the number of automatically constructed temporal and statistical features being used in the RIPPER rules. We see that for both the "traffic" and host-based "traffic" models, our feature construction process contributes the majority of the features actually used in the rules.

\subsection{Off-line Detection Results}

We report here the performance of our detection models as evaluated by MIT Lincoln Lab. We trained our intrusion detection models, i.e., the base models and the meta-level classifier, using the 7 weeks of labeled data, and used them to make predictions on the 2 weeks of unlabeled test data. The test data contains a total of 38 attack types, with 14 types in the test data only (i.e., our models were not trained with instances of these attack types).

Figure 3 shows the ROC curves of the detection models by attack category as well as on all intrusions. In each of these ROC plots, the $\mathrm{x}$-axis is the false alarm rate, calculated as the percentage of normal connections classified as an intrusion; the $y$-axis is the detection rate, calculated as the percentage of intrusions detected (since the models produced binary outputs, the ROC curves are not continuous). We compare here our models with other participants (denoted as Group 1 to 3 ) in the DARPA eval- 


\begin{tabular}{|llllll|}
\hline Model & Feature set & $\begin{array}{l}\text { Intrusion } \\
\text { categories }\end{array}$ & $\begin{array}{l}\text { \# of features } \\
\text { in records }\end{array}$ & \# of rules & $\begin{array}{l}\text { \# of features } \\
\text { used in rules }\end{array}$ \\
\hline content & $\begin{array}{l}\text { "intrinsic" } \\
\text { "content" }\end{array}$ & U2R, R2L & 22 & 55 & 11 \\
traffic & $\begin{array}{l}\text { "intrinsic" + } \\
\text { "traffic" }\end{array}$ & DOS, PROBING & 20 & 26 & $4+\mathbf{9}$ \\
\hline & $\begin{array}{l}\text { "intrinsic" + } \\
\text { "host traffic" }\end{array}$ & Slow PROBING & 14 & 8 & $1+\mathbf{5}$ \\
\hline
\end{tabular}

Table 6. Model Complexities

uation program (these plots are duplicated from the presentation slides of a report given by Lincoln Lab in a DARPA PI meeting. The slides can be viewed on line via http://www.cs.columbia.edu/ sal/JAM/ PROJECT/MIT/mit-index.html). These groups primarily used knowledge engineering approaches to build their intrusion detection systems. We can see from the figure that our detection models have the best overall performance, and in all but one attack category, our model is one of the best two.

\section{Formalizing Cost-based Models for Intru- sion Detection}

In the credit card fraud domain, the notion of costs is inextricably intertwined with the learning task. We seek to learn models of fraudulent transactions that minimizes the overall loss. We believe an analogous cost optimization problem can and should be defined for the intrusion detection system (IDS) domain.

In the arena of IDS, there are at least three types of costs involved (that are derivative of the credit card fraud case):

1. "Damage" cost: the amount of damage caused by an attack if intrusion detection is not available or an IDS fails to detect an attack;

2. "Challenge" cost: the cost to act upon a potential intrusion when it is detected; and

3. "Operational" cost: the resources needed to run the IDS.

Table 7 illustrates our perspective on the three types of cost in credit card fraud and intrusion detection. In the credit card case, "damage" is the amount of a fraudulent transaction that the bank losses, $\operatorname{tranamt}(t)$. In the IDS case, damage can be characterized as a function that depends on the type of service and attack on that service, DCost (service, attack). The challenge cost for both cases is term as overhead, which is the cost of acting on an alarm. We did not consider operational cost in the credit card case because we did not have the opportunity to study this aspect of the problem. The banks have existing fielded systems whose total aggregated operational costs have already been considered and are folded into their overhead costs (here called the challenge cost). We shall take a limited view of this by considering the costs of alternative models based upon the "feature costs" used by these models employed in an IDS and we denote this operational cost as OpCost. We next elaborate on each of these sources of cost.

\subsection{Damage costs}

The damage cost characterizes the amount of damage inflicted by an attack when intrusion detection is unavailable (the case for most systems). This is important and very difficult to define since it is likely a function of the particulars of the site that seeks to protect itself. The defined cost function per attack or attack type should be used here to measure the cost of damage. This means, that rather than simply measuring $F N$ as a rate of missed intrusions, rather we should measure total loss based upon $D \operatorname{Cost}(s, a)$, which varies with the service $(s)$ and the specific type of attack $(a)$. These costs are used throughout our discussion.

\subsection{Challenge costs}

The challenge cost is the cost to act upon an alarm that indicates a potential intrusion. For IDS, one might consider dropping or suspending a suspicious connection and attempting to check, by analyzing the service request, if any system data have been compromised, or system resources have been abused or blocked from other legitimate users. (Other personnel time costs can be folded in including gathering evidence for prosecution purposes if the intruder can be traced.) These costs can be estimated, as a first cut, by the amount of CPU and disk resources needed to challenge a suspicious connection. For simplicity, instead of estimating the challenge cost for each intrusive connection, we can "average" (or amortize over a large volume of connections during some standard "business cycle") the challenge costs to a single (but not static) challenge cost per potential intrusive connection, i.e., overhead. 


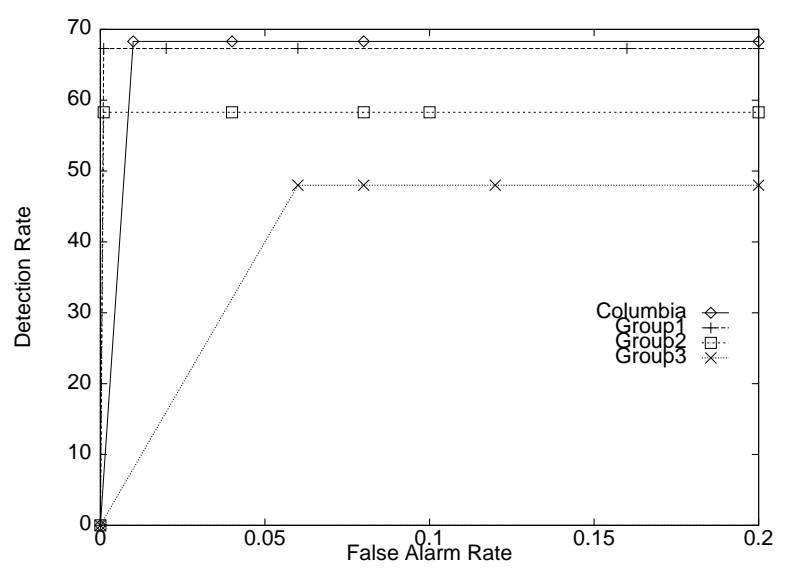

(a) DOS

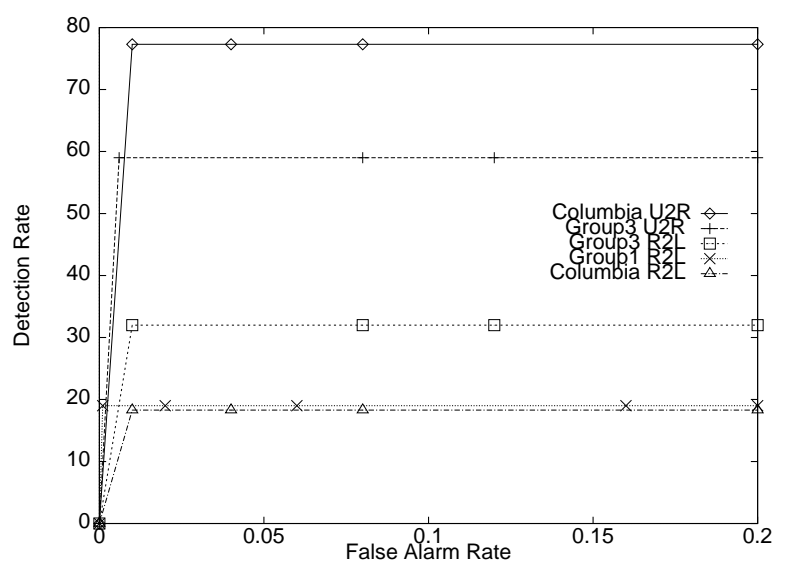

(c) U2R and R2L

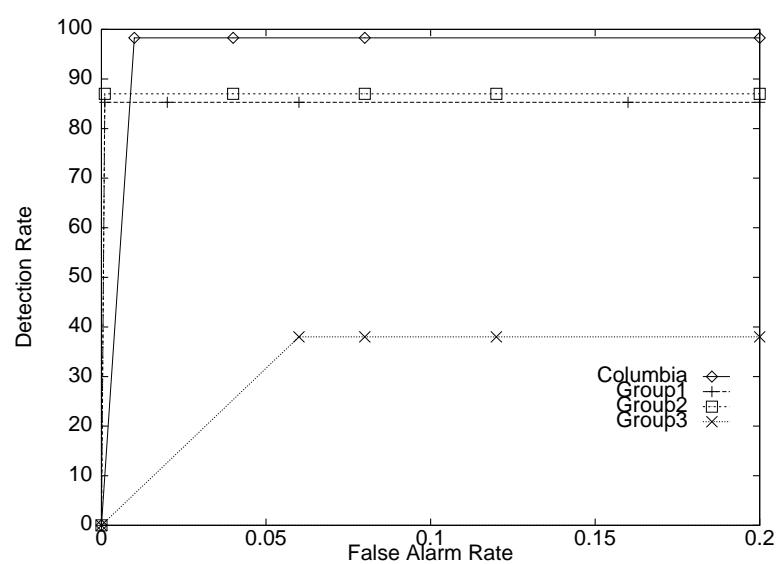

(b) PROBING

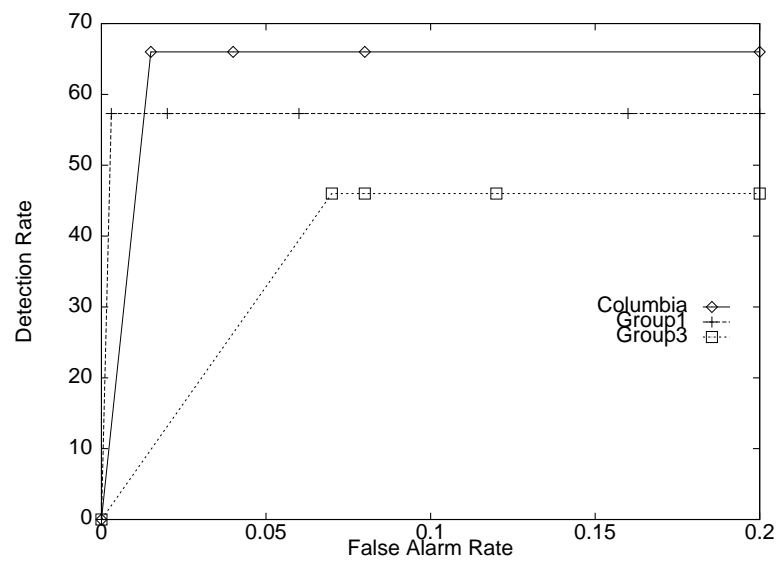

(d) Overall

Figure 3. ROC Curves on Detection Rates and False Alarm Rates

\subsection{Operational costs}

The cost of fielding a detection system is interesting to consider in some detail. In the work on fraud detection in financial systems, we learned that there are a myriad of "business costs" involved in design, engineering, fielding and use (challenge) of detection systems. Each contributes to an overall aggregated cost of detecting fraud. The main issue in operational costs for IDS is the amount of resources to extract and test features from raw traffic data. Some features are costlier than others to gather, and at times, costlier features are more informative for detecting intrusions.

Real-time constraints in IDS. Even if one designs a good detection system that includes a set of good features that well distinguish among different attack types, these features may be infeasible to compute and maintain in real time. In the credit card case, transactions have a 5 second response constraint (a desired average waiting time). That's a lot of time to look up, update and compute and test features, per transaction. In the IDS case, the desired average response rate should be measured in terms of average connection times, or even by TCP packet rates, a much smaller time frame, so connections can be dropped as quickly as possible before they do damage.

In the case of IDS it is not obvious when an intrusion can be detected, and when an alarm should be issued. Ideally, we would like to detect and generate an alarm during an ongoing attack connection in order to disable it, rather than after the fact when damage has already been done. However, certain models of intrusive connections may require information only known at the conclusion of a connection! Thus, properly designing an intrusion detection system requires that considerable thought be given to the time at which a detection can and should take place.

Therefore, although the problems appear quite similar, 


\begin{tabular}{|c|c|c|}
\hline Cost Type & Credit Card Fraud & Network Intrusion \\
\hline Damage & tranamt $(t)$ & DCost( service, attack) \\
Challenge & overhead & overhead \\
Operational & subsumed in overhead & OpCost \\
\hline
\end{tabular}

Table 7. Cost types in credit card fraud and network intrusion

the constraints are really much different between the two task domains. The problem seems to be much harder in the IDS case since we have to accommodate in our cost models the response rate of the system. It seems evident that a slower IDS should be penalized with a higher cost. (In the credit card case we simply ignored this cost.) This important source of cost however is a major topic of research for IDS, i.e. the computational costs for rapid detection. Our work in this area is new and ongoing. Details of our initial thoughts here can be found in [13].

\subsection{Cost Model for IDS}

We just described the three different types of cost in IDS: damage cost, challenge cost, and operational cost. Our cost model for IDS considers these three types of cost. Similar to the credit card case, the IDS cost model depends on the outcomes of the IDS' predictions: false negative (FN), false positive (FP), true positive (TP), and true negative (TN). We now examine the cost associated with each of these outcomes.

FN cost, or the cost of NOT detecting an attack, is the most dangerous case (and is incurred by most systems today that do not field IDS's). Here, the IDS "Falsely" decides that a connection is not an attack and there is no challenge against the attack. This means the attack will succeed and do its dirty work and presumably some service will be lost, and the organization losses a service of some value. The FN Cost is, therefore, defined as the damage cost associated with the particular type of service and attack, $D \operatorname{Cost}(s, a)$.

TP Cost is the cost of detecting an attack and doing something about it, i.e. challenging it. Here, one hopes to stop an attack from losing the value of the service. There is a cost of challenging the attack, however, that is involved here. When some event triggers an IDS to correctly predict that a True attack is underway (or has happened), then what shall we do? If the cost to challenge the attack is overhead, but the attack affected a service whose value is less than overhead, then clearly ignoring these attacks saves cost. Therefore, for a true positive, if overhead $>D \operatorname{Cost}(s, a)$, the intrusion is not challenged and the loss is $D \operatorname{Cost}(s, a)$, but if overhead $<\operatorname{DCost}(s, a)$, the intrusion is challenged and the loss is limited to overhead.

FP cost. When an IDS falsely accuses an event of being an attack, and the attack type is regarded as high cost, a challenge will ensue. We pay the cost of the challenge (overhead), but nothing really happened bad except we lost overhead on the challenge. Naturally, when evaluating an IDS we have to concern ourselves with measuring this loss. For this discussion, we define the loss is just overhead for a false positive.

$T N$ cost. An IDS correctly decides that a connection is normal and Truly not an attack. We therefore bare no cost that is dependent on the outcome of an IDS.

Thus far we have only considered costs that depend on the outcome of an IDS, we now incorporate the operational cost, OpCost, that is independent of the IDS' predictive performance. Our notion of OpCost mainly measures the cost of computing values of features in the IDS. We denote $O p$ $\operatorname{Cost}(c)$ as the operational cost for a connection, $c$.

We now can describe the cost-model for IDS. When evaluating an IDS over some test set $S$ of labeled connections, $c \in S$, we define the cumulative cost for a detector as follows:

$$
\text { CumulativeCost }(S)=\sum_{c \in S}(\operatorname{Cost}(c)+O p \operatorname{Cost}(c))
$$

where $\operatorname{Cost}(c)$ is defined (analogous to the credit card case) in Table 8. Here $s$ is the service requested by connection $c$ and $a$ is the attack type detected by the IDS for the connection.

Note that a higher operational cost, $O p \operatorname{Cost}(c)$, could be incurred by employing "expensive" features; but this may potentially improve the predictive performance of the IDS and thus lower $\operatorname{Cost}(c)$. Hence, in order to minimize Cumulative $\operatorname{Cost}(S)$, we need to investigate and quantify, in realistic contexts, the trade off between $\operatorname{OpCost}(c)$ and $\operatorname{Cost}(c)$ in Equation 1. This issue constitutes a major part of our ongoing research in the JAM project.

\subsection{Flying Under Mobile Radar: Dynamic Over- head Adjustment}

As in the credit card case, we can simplify the IDS cost model by subsuming the operational costs into overhead (challenge cost). In this way the cumulative cost of an IDS is highly dependent upon the overhead ${ }^{10}$ value set at

\footnotetext{
${ }^{10}$ and we may regard the overhead as the minimum height that a radar system protecting an area can see. Cruise missile programmers have an
} 


\begin{tabular}{|c|c|c|}
\hline Outcome & $\operatorname{Cost}(c)$ & \\
\hline Miss (False Negative, $F N$ ) & $D \operatorname{Cost}(s, a)$ & \\
\hline False Alarm (False Positive, $F P$ ) & $\begin{array}{l}\text { overhead } \\
0\end{array}$ & $\begin{array}{l}\text { if } D \operatorname{Cost}(s, a)>\text { overhead or } \\
\text { if } D \operatorname{Cost}(s, a) \leq \text { overhead }\end{array}$ \\
\hline Hit (True Positive, $T P$ ) & $\begin{array}{l}\text { overhead } \\
\text { DCost }(s, a)\end{array}$ & $\begin{array}{l}\text { if } D \operatorname{Cost}(s, a)>\text { overhead or } \\
\text { if } D \operatorname{Cost}(s, a) \leq \text { overhead }\end{array}$ \\
\hline Normal (True Negative, $T N$ ) & 0 & \\
\hline
\end{tabular}

Table 8. Cost Model for Connection

the time models are computed, and certainly when they are evaluated. It is quite possible, and virtually assured, that under different overheads, different models will be computed and different cost performance will be exhibited.

In some industries (not only the credit card industry), overheads are so firmly fixed that fraud is simply modeled as another "cost of doing business" and is simply tolerated $^{11}$. The overhead amount is defined by a myriad of business costs, but it need not be static when applied at run-time! Thus, it is quite logical to vary the overhead limit when operating an IDS, thus changing the challenge cost producing different behavior and cost performance of the detection system. This simple strategy tends to enter "noise" making it difficult for perpetrators to "optimize" their thefts.

But notice that under a changing overhead, either up or down, for which detectors had originally been trained, the outcome of cost savings attributed to the detector might vary widely. This change in overhead has another fundamental effect: it changes the environment from which our underlying distribution is drawn. This means, that once we lower the overhead, thieves might learn to lower their appetite for stealing not to get caught. Concurrently, raising the overhead afterwards might then generate large cost savings, until the thieves have learned to return to their former ways of being greedy.

An interesting question, therefore, is whether there is an optimal strategy of dynamically varying the overhead in order to maximize savings over a longer period of time. Varying the overhead implies that we must concern ourselves with potentially "non-linear effects" in cost savings. A slight reduction may indeed catch more fraud, but may result in far heavier losses due to the real costs of challenging a new found wealth of "cheap fraud"!

exceptionally interesting task of assuring their flying missiles stay below this radar to deliver their ordinance!

${ }^{11}$ For example, in the auto insurance industry, broken windshields are regarded as an immediately approved expense. Fraud perpetrators will submit insurance charges for bogus repairs of windshields and be assured of payment, simply because the cost of investigation is prohibitively expensive. Here thieves have a different problem. They need to learn the rate at which they submit bogus claims not to draw obvious attention to themselves from human claims processing personnel, the low bandwidth, final detectors.
This begs further questions and deeper study to determine alternative strategies. Perhaps classifiers ought to be entirely retrained, or meta-classifiers might re-weight their constituent base classifiers under a new changing fraud and cost distribution, and when should we do this? Or, simply measuring daily cost savings performance, or the rate of change thereof, might provide interesting clues to an optimal daily setting? The rate at which we change our overhead setting, and/or our models to avoid widely varying oscillations in performance of overall cost savings is not obvious.

It is interesting to note here that one of the design goals of JAM is to provide a scalable, efficient and hence adaptable distributed learning system that provides the means of rapidly learning new classifiers, and distributing (via agent architectures) new detectors to accommodate changing conditions of the environment in which it operates. Another avenue for exploration in JAM is therefore to perhaps dynamically re-weight ensembles of classifiers, our metaclassifiers, to adjust to new overhead limits.

\subsection{Summary}

In our work on intrusion detection, the data mining activity was focussed on uncovering likely features to extract from the streaming TCP packets preprocessed into connection records that are used in preparing training data, computing models and testing those models.

However, much of the traditional research in modeling only considers statistical accuracy, or $T P / F P$ rates of models when comparing approaches. We should now understand that accuracy is not the whole picture. In different real world contexts, "cost" can take on different meanings, and the target application might necessarily be defined as a cost optimization problem.

In the context of IDS, real time performance is crucial. Here cost measures involve throughput and memory resources. It is of no value if one has an IDS that consumes so much resource that services can no longer be delivered on time, or the cost of fielding the IDS is so high that it becomes uneconomical to do so. 


\section{Conclusion}

In this paper, we presented the main results of the JAM project. We focused the discussion on cost-sensitive modeling techniques for credit card fraud detection and network intrusion detection. We showed that the models built using our distributed and cost-sensitive learning techniques can yield substantial cost savings for the financial institutions. We reported our research in applying data mining techniques to build intrusion detection models. The results from the 1998 DARPA Intrusion Detection Evaluation showed that our techniques are very effective. We briefly examined the cost factors and cost models in intrusion detection, and discussed the challenges in cost-sensitive modeling for intrusion detection.

\subsection{Future Work}

There a number of open research issues that need to be addressed in the general setting of distributed data mining, but also specific to the important task of detecting intrusions:

1. How does an organization or domain rationally set the costs of its various services and systems it wishes to protect with an IDS, thus defining $\operatorname{Cost}(s, a)$ for all services and all attack types? And how do we rationally determine an overhead challenge cost, overhead especially under tough real-time constraints?

2. What "cost sensitive" data mining and machine learning algorithms are needed to generate "low cost" models; i.e. models that are cheap to evaluate and operate under (variable) "real-time" constraints, and that also maximize cost savings or minimize loss?

3. Specifically for network-based intrusion detection, what is the optimal set of features to best model a "good detector" for different environments and platforms?

4. The distribution of attacks, and the various costs associated with services and attacks will naturally change over time. What adaptive strategies might be needed to optimally change models or mixtures of models to improve detection and at what rate of change?

5. Likewise, what strategies may be employed in dynamically adjust overhead challenge costs (overhead) to maximize cost savings for a fixed detection system over larger time periods.

In conclusion, we need a microeconomic theory of intrusion detection.

\section{Acknowledgments}

This research is supported in part by grants from DARPA (F30602-96-1-0311) and NSF (IRI-96-32225 and CDA-9625374).

Our work has benefited from in-depth discussions with Alexander Tuzhilin of New York University and Foster Provost of Bell Atlantic research, and suggestions from Charles Elkan of UC San Diego.

\section{References}

[1] R. Agrawal, T. Imielinski, and A. Swami. Mining association rules between sets of items in large databases. In Proceedings of the ACM SIGMOD Conference on Management of Data, pages 207-216, 1993.

[2] R. Agrawal and R. Srikant. Fast algorithms for mining association rules. In Proceedings of the 20th VLDB Conference, Santiago, Chile, 1994.

[3] P. Chan and S. Stolfo. Meta-learning for multistrategy and parallel learning. In Proc. Second Intl. Work. Multistrategy Learning, pages 150-165, 1993.

[4] P. Chan and S. Stolfo. Toward scalable and parallel learning: A case study in splice junction prediction. Technical Report CUCS-032-94, Department of Computer Science, Columbia University, New York, NY, 1994. (Presented at the ML94 Workshop on Machine Learning and Molecular Biology).

[5] P. Chan and S. Stolfo. Scaling learning by metalearning over disjoint and partially replicated data. In Proc. Ninth Florida AI Research Symposium, pages 151-155, 1996.

[6] P. Chan and S. Stolfo. Sharing learned models among remote database partitions by local meta-learning. In Proc. Second Intl. Conf. Knowledge Discovery and Data Mining, pages 2-7, 1996.

[7] P. Chan and S. Stolfo. Learning with non-uniform distributions: Effects and a multi-classifier approach. Submitted to Machine Learning Journal, 1999.

[8] W. W. Cohen. Fast effective rule induction. In Machine Learning: the 12th International Conference, Lake Taho, CA, 1995. Morgan Kaufmann.

[9] W. Fan, S. Stolfo, and J. Zhang. Adacost: Misclassification cost-sensitive boosting. In Proceedings Internation Conference on Machine Learning, 1999. 
[10] W. Lee and S. J. Stolfo. Data mining approaches for intrusion detection. In Proceedings of the 7th USENIX Security Symposium, San Antonio, TX, January 1998.

[11] W. Lee, S. J. Stolfo, and P. K. Chan. Learning patterns from unix process execution traces for intrusion detection. In AAAI Workshop: AI Approaches to Fraud Detection and Risk Management, pages 50-56. AAAI Press, July 1997.

[12] W. Lee, S. J. Stolfo, and K. W. Mok. A data mining framework for building intrusion detection models. In Proceedings of the 1999 IEEE Symposium on Security and Privacy, May 1999.

[13] W. Lee, S. J. Stolfo, and K. W. Mok. Mining in a data-flow environment: Experience in network intrusion detection. In Proceedings of the ACM SIGKDD International Conference on Knowledge Discovery \& Data Mining (KDD-99), August 1999.

[14] Jacek Maitan, Zbigniew W. Ras, and Maria Zemankova. Query handling and learning in a distributed intelligent system. In Zbigniew W. Ras, editor, Methodologies for Intelligent Systems, 4, pages 118-127, Charlotte, North Carolina, October 1989. North Holland.

[15] H. Mannila and H. Toivonen. Discovering generalized episodes using minimal occurrences. In Proceedings of the 2nd International Conference on Knowledge Discovery in Databases and Data Mining, Portland, Oregon, August 1996.

[16] H. Mannila, H. Toivonen, and A. I. Verkamo. Discovering frequent episodes in sequences. In Proceedings of the 1st International Conference on Knowledge Discovery in Databases and Data Mining, Montreal, Canada, August 1995.

[17] M. Nunez. Economic induction: A case study. In Proceedings of the Third European Working Session on Learning, EWSL-88, pages 139-145. Morgan Kaufmann, 1988.

[18] A. L. Prodromidis and S. J. Stolfo. Mining databases with different schemas: Integrating incompatible classifiers. In G. Piatetsky-Shapiro R Agrawal, P. Stolorz, editor, Proc. 4th Intl. Conf. Knowledge Discovery and Data Mining, pages 314-318. AAAI Press, 1998.

[19] A. L. Prodromidis and S. J. Stolfo. Agent-based distributed learning applied to fraud detection. In Sixteenth National Conference on Artificial Intelligence, 1999. Submitted for publication.
[20] A.L. Prodromidis and S.J. Stolfo. A comparative evaluation of meta-learning strategies over large and distributed data sets. In Workshop on Meta-learning, Sixteenth Intl. Conf. Machine Learning, 1999. Submitted for publication.

[21] Zbigniew W. Ras. Answering non-standard queries in distributed knowledge-based systems. In L. Polkowski A. Skowron, editor, Rough sets in Knowledge Discovery, Studies in Fuzziness and Soft Computing, volume 2, pages 98-108. Physica Verlag, 1998.

[22] R. Schapire and Y. Singer. Improved boosting algorithms using confidence-rated predictions. In Proceedings of the Eleventh Annual Conf on Computational Learning Theory, 1998.

[23] StatSci Division, MathSoft, Seattle. Splus, Version 3.4, 1996.

[24] S. Stolfo, W. Fan, W. Lee, A. Prodromidis, and P. Chan. Credit card fraud detection using metalearning: Issues and initial results. Working notes of AAAI Workshop on AI Approaches to Fraud Detection and Risk Management, 1997.

[25] S. Stolfo, W.D. Fan, A. Prodromidis W.Lee, S. Tselepis, and P. K. Chan. Agentbased fraud and intrusion detection in financial information systems. Available from http://www.cs.columbia.edu/ sal/JAM/PROJECT, 1998.

[26] S. Stolfo, A. Prodromidis, S. Tselepis, W. Lee, W. Fan, and P. Chan. JAM: Java agents for meta-learning over distributed databases. In Proc. 3rd Intl. Conf. Knowledge Discovery and Data Mining, pages 74-81, 1997.

[27] M. Tan and J. Schlimmer. Two case studies in costsensitive concept acquition. In Proceedings of the Eight National Conference on Artificial Intelligence, pages 854-860, Boston, MA, 1990.

[28] P. D. Turney. Cost-sensitive classification: Empirical evaluation of a hydrid genetic decision tree induction algorithm. Journal of AI Research, 2:369-409, 1995.

[29] D. Wolpert. Stacked generalization. Neural Networks, 5:241-259, 1992. 